\title{
Atuação profissional dos estomaterapeutas egressos da Universidade Federal de Minas Gerais
}

\section{Professional activity of stomatherapists graduated from the Federal University of Minas Gerais}

\section{Actuación profesional de los estomaterapeutas egresados de la Universidad Federal de Minas Gerais}

\author{
Thiago Gomes Gontijo ${ }^{1 *}$, Eline Lima Borges², Aidê Ferreira Ferraz², José Ferreira Pires Júnior ${ }^{2,3}$, \\ Josimare Aparecida Otoni Spira²
}

ORCID IDS

Gontijo TG (D) https://orcid.org/0000-0002-7582-275X

Borges EL (D) https://orcid.org/0000-0002-0623-5308

Ferraz AF (D) https://orcid.org/0000-0002-0610-3946

Pires Júnior JF (iD https://orcid.org/0000-0002-6019-0198

Spira JAO (D) https://orcid.org/0000-0002-6577-0394

\section{COMO CITAR}

Gontijo TG; Borges EL; Ferraz AF; Pires Júnior JF; Spira JAO (2019). Atuação profissional dos estomaterapeutas egressos da Universidade Federal de Minas Gerais. ESTIMA, Braz. J. Enterostomal Ther., 17: e1419. https://doi.org/10.30886/estima.v17.686_PT

\begin{abstract}
RESUMO
Objetivos: Este estudo teve como objetivos identificar a área de atuação profissional, caracterizar a trajetória e a prática clínica na área da estomaterapia e identificar posteriores capacitações como especialistas. Método: Estudo descritivo com abordagem quantitativa realizado por meio da aplicação de questionário online aos egressos do curso de especialização em Estomaterapia da Universidade Federal de Minas Gerais, iniciado no ano de 2007, totalizando 97 egressos até 2015. A amostra por conveniência foi composta pelos 84 especialistas que concordaram em responder o questionário contemplando as variáveis do estudo. Resultados: Os egressos eram em sua maioria do sexo feminino (86\%), com idade entre 31 e 40 anos (68\%), residentes (58\%) e atuantes (59,6\%) em Belo Horizonte. Mantêm-se atuantes em sua área de especialidade (70\%), com predominância dos cuidados com estomias e feridas (31\%), em atividades assistenciais (29,3\%), na área hospitalar (27,3\%) e no setor público de saúde (38\%). A busca pela especialização foi motivada predominantemente pelo interesse no desenvolvimento técnico-científico (48,5\%) e (69\%) dos egressos continua se capacitando após a especialização. Conclusão: A estomaterapia encontra-se em expansão no mercado nacional, sendo necessária a continuidade de pesquisas de âmbito nacional para uma visão mais abrangente da especialidade.
\end{abstract}

DESCRITORES: Área de atuação profissional;Enfermagem; Especialidades de enfermagem; Estomaterapia.

1.Centro Universitário Estácio de Belo Horizonte - Departamento de Saúde - Belo Horizonte/MG - Brasil.

2.Universidade Federal de Minas Gerais - Escola de Enfermagem - Programa de Pós-graduação em Enfermagem, Belo Horizonte/MG - Brasil.

3.Instituto Mário Penna - Hospital Luxemburgo - Serviço de Estomaterapia, Belo Horizonte/MG - Brasil.

Autor correspondente: thiago.gontijo15@gmail.com

Recebido: Dec. 26, 2018 | Aceito: Ago. 28, 2019 


\begin{abstract}
Objectives: This study aimed to identify the area of professional activity, characterize the trajectory and clinical practice in the area of stomatherapy, and identify further training as a specialist. Method: A descriptive study with a quantitative approach was conducted by applying an online questionnaire to graduates of the specialization course in Stomatherapy of the Federal University of Minas Gerais, which began in 2007, totaling 97 by 2015. The convenience sample was composed of 84 specialists who agreed to answer the questionnaire considering the study variables. Results: Most of the graduates were female (86\%), aged between 31 and 40 years (68\%), residents (58\%) and working (59.6\%) in Belo Horizonte. They remain active in their specialty area (70\%), with predominance of stoma and wound care (31\%), in care activities (29.3\%), in the hospital area (27.3\%) and in the health public sector (38\%). The pursuit of specialization was predominantly motivated by interest in technical-scientific development (48.5\%) and (69\%) of the graduates continues capacitating after specialization. Conclusion: Stomatherapy is expanding in the national market, requiring the continuation of national research for a broader view of the specialty.
\end{abstract}

DESCRIPTORS: Professional practice area; Nursing; Nursing specialties; Stomatherapy.

\title{
RESUMEN
}

Objetivos: Este estudio pretendió identificar el área de actuación, caracterizar la trayectoria y la práctica clínica en el área de la estomaterapia e identificar subsiguientes capacitaciones como experto. Método: Estudio descriptivo con enfoque cuantitativo llevado a cabo mediante la aplicación de un cuestionario en línea a los egresados del Curso de Especialización en Estomaterapia de la Universidad Federal de Minas Gerais, iniciado en el año 2007, totalizando 97 hastas el año 2015. La muestra de coveniencia consistió em 84 expertos que aceptaron responder el cuestionario considerando las variables del estúdio. Resultados: La mayoría de los egresados eran mujeres (86\%), con una edad comprendida entre los 31 y los 40 años (68\%), residentes (58\%) y actuantes (59,6\%) en Belo Horizonte. Se mantienen actuantes en el área de especialidad (70\%), con predominio de la atención a las estomías y las heridas (31\%), en actividades de asistencia (29,3\%), en el área hospitalaria (27,3\%) y en el sector público de salud (38\%). La búsqueda de especialización estuvo motivada, predominantemente, por el interés en el desarrollo técnico-científico (48,5\%) y (69\%) de los egresados siguen siendo entrenados post-especialización. Conclusión: Se concluye que la estomaterapia se está expandiendo en el mercado brasileño, lo que requiere la continuidad de investigaciones nacionales para una visión más amplia de esta especialidad.

DESCRIPTORES: Ubicación de la práctica profesional; Enfermería; Especialidades de enfermería; Estomaterapia.

\section{INTRODUÇÃO}

Os cursos de especialização em Enfermagem propõem e propiciam ao profissional atualização e especialização do conhecimento específico, na execução de atividades assistenciais, administrativas, gerenciais, de ensino, pesquisa e integração. Para o enfermeiro, é imprescindível aprimorar a prática do cuidado ao cliente, à família e à comunidade, em qualquer contexto, e que atenda não somente às expectativas do sistema de saúde, mas principalmente às reais necessidades da populaçãa ${ }^{1}$.

O enfermeiro especialista em estomaterapia ou estomaterapeuta é o profissional que detém conhecimentos técnicos, capacitação específica e competências para prestar assistência às pessoas com estomias, feridas agudas e crônicas, fístulas e incontinência anal e urinária em ações preventivas, terapêuticas e de reabilitação em busca de melhor qualidade de vida ${ }^{2,3}$.

O processo de formação do estomaterapeuta, segundo a Associação Brasileira de Estomaterapia (Sobest), exige que o enfermeiro realize um curso de especialização em Enfermagem em Estomaterapia, que tenha abrangência integral nas áreas da especialidade (estomias, feridas, fístulas e incontinência anal e urinária), seja reconhecido pelos órgãos nacionais de educação, pela Sobest e credenciados no World Council of Enterostomal Therapists (WCET) ${ }^{2}$.

A estomaterapia obteve destaque mundial em 1978, com a criação de um órgão internacional representativo, $o$ WCET, que tem como objetivo promover a identidade da estomaterapia e favorecer a troca de informações entre os profissionais, além de contribuir para o desenvolvimento técnico e científico da especialidade, a uniformização das ações com vistas a um melhor desempenho da estomaterapia e, consequentemente, a qualidade da assistência prestada à pessoa com estomia, bem como à pessoa com feridas e incontinência ${ }^{3}$.

No Brasil, a estomaterapia teve início formalmente em 1990 com a introdução do curso de especialização em Enfermagem em Estomaterapia na Escola da Universidade de São Paulo ${ }^{4}$. 
Outro marco considerável na história da estomaterapia brasileira foi a fundação da Sociedade Brasileira de Estomaterapia (Sobest) em dezembro de 1992. A partir de 1997, é renomeada como Associação Brasileira de Estomaterapia: estomias, feridas e incontinências, por não ter fins lucrativos. Em 2003, a Sobest criou a Revista Estima, ferramenta oficial de difusão da estomaterapia que trata dos conhecimentos científicos e atuais dos campos da especialidade ${ }^{4}$.

A Escola de Enfermagem da Universidade Federal de Minas Gerais (EEUFMG) foi pioneira no estado de Minas Gerais ao criar o primeiro curso de especialização em estomaterapia. Desde suas primeiras turmas, o curso tem registro na Sobest e no WCET.

O curso teve início na EEUFMG no ano de 2007, com uma média de 13 vagas ofertadas por ano. Os objetivos do curso são proporcionar ao enfermeiro embasamento para aprofundar conhecimentos específicos e desenvolver sua competência técnica, pedagógica, gerencial e investigativa. É um curso com proposta pedagógica inovadora que visa atender a diversidade de demandas do mercado de trabalho na área, correspondendo aos avanços da enfermagem enquanto ciência e às diversas necessidades de saúde da população ${ }^{2}$.

Ao se considerar os marcos históricos do curso da UFMG e sua abrangência teórico-prática para os profissionais após a formação de oito turmas, fez-se necessário conhecer o fazer desses especialistas enquanto agentes de multiplicação do saber e de transformação social.Para a continuidade das mudanças é essencial a identificação da realidade dos egressos do curso de especialização em Enfermagem em Estomaterapia; por isso, surgiu o interesse em estudar esse tema.

Os resultados deste estudo irão contribuir para o dimensionamento e mapeamento da inserção dos estomaterapeutas no mercado de trabalho, embasamento para revisar a grade curricular dos cursos de especialização, além de identificar lacunas no conhecimento que irão favorecer estudos posteriores e complementares. Poderão ainda possibilitar a avaliação das oportunidades de inserção do estomaterapeuta na prática da especialidade.

\section{OBJETIVOS}

Identificar a área de atuação dos estomaterapeutas egressos da UFMG, caracterizar a trajetória e prática clínica quanto à área de atuação específica na estomaterapia e identificar posteriores capacitações na formação como especialistas na área.

\section{MÉTODOS}

Trata-se de estudo descritivo com abordagem quantitativa abrangendo participantes dos estados de Minas Gerais, Bahia, Espírito Santo, São Paulo e Mato Grosso do Sul, egressos do curso de especialização em Estomaterapia da EEUFMG.

No período de 2007 a 2015, o curso da UFMG formou oito turmas, totalizando 97 especialistas. Para a composição da amostra, os egressos foram convidados a participar do estudo por meio de carta enviada por correio eletrônico. Após um mês, a equipe recebeu o aceite de 84 especialistas $(86,5 \%)$ que fizeram parte da amostra, por conveniência.

A coleta de dados ocorreu no período de março a abril de 2017 e demandou a elaboração de um questionário, por meio do aplicativo Google Forms composto por 15 questões que contemplaram variáveis sociodemográficas (nome, idade, sexo, cidade em que reside, cidade em que trabalha, ano de conclusão do curso de graduação e ano de conclusão do curso de especialização em Estomaterapia) e perfil profissional dos especialistas (áreas de atuação após conclusão da pós-graduação).

Inicialmente, os participantes receberam o link via correio eletrônico para acesso e preenchimento do questionário. Após 15 dias, o link foi reenviado, também via correio eletrônico, para os participantes que ainda não haviam respondido o questionário.

O desenvolvimento da pesquisa respeitou os preceitos éticos estabelecidos pela Resolução no 466, de 12 de dezembro de 2012. O estudo foi aprovado pelo Comitê de Ética em Pesquisa da UFMG (CAAE 64685417.7.0000.5149) sob o parecer $n^{\circ} 2.022 .019$. Os participantes, que após esclarecimentos e explicação do desenvolvimento da pesquisa concordaram em participar do estudo, assinaram o Termo de Consentimento Livre e Esclarecido (TCLE) e o enviaram digitalizado via correio eletrônico. $\mathrm{O}$ anonimato e a ausência de ônus financeiro foram garantidos aos participantes.

Os dados obtidos foram digitados em planilhas do programa Microsoft Office Excel, versão 2007. A seguir, 
as tabelas geradas foram submetidas a análise descritiva e os resultados discutidos à luz da literatura.

\section{RESULTADOS}

A amostra constituiu-se de 84 egressos do curso de especialização em Estomaterapia da EEUFMG, que correspondem a $86,5 \%$ do número total de formandos do curso no período de 2007 a 2015. A apresentação das variáveis sociodemográficas dessa amostra encontra-se na Tabela 1.

$\mathrm{Na}$ caracterização quanto ao sexo, prevaleceu o gênero feminino $(85,7 \%)$. A maioria dos participantes tem faixa etária entre 31 a 40 anos $(67,8 \%)$, seguida de 41 a 50 anos $(13,1 \%)$, e de 20 a 30 anos (8,3\%), com idade mínima de 27 anos, idade máxima de 66 anos e média de 38,4 anos.

Dos egressos, 49 (58,3\%) residem em Belo Horizonte/MG, 22 (26,2\%) em cidades do interior de Minas Gerais, 8 (9,5\%) em Contagem/MG e 1 (1,2\%) em Betim/MG. Identificou-se que 4 (4,7\%) egressos residem em outros estados: 1 (1,2\%) em Ponta Porã/MS, 1 (1,2\%) em São Paulo/SP, 1 (1,2\%) em Teixeira de Freitas/BA e 1 (1,2\%) em Vitória/ES.

Referente ao local de trabalho, 50 egressos (59,5\%) trabalham em Belo Horizonte, 19 (22,6\%) em cidades no interior de Minas Gerais, 4 (4,7\%) em Contagem/MG, 3 (3,6\%) em Betim/MG, 4 (4,7\%) em outros estados (Bahia, Espírito Santo, Mato Grosso e São Paulo) e 2 (2,4\%) egressos trabalham em mais de um estado. No momento, 2 (2,3\%) egressos estão desempregados.

Em relação ao ano de conclusão do curso de graduação, prevaleceram concluintes entre os anos de 2006 a 2010, com 36 egressos (42,8\%); e $24(28,5 \%)$ entre os anos de 2001 a 2005.

Dos 84 egressos do curso de Estomaterapia que participaram da pesquisa, a predominância do ano de conclusão do curso foi de 19 (22,6\%) concluintes em 2015, seguido de 12 (14,3\%) em 2009, 12 (14,3\%) em 2014, 11 $(13,1 \%)$ em 2012, 10 (11,9\%) em 2010, 7 (8,3\%) em 2013, $5(6,0 \%)$ em 2008, 8 (9,5\%) em 2007 e 8 (9,5\%) em 2011.

Dos 84 participantes da pesquisa, 73 (86,9\%) atuam como enfermeiros e $11(13,1 \%)$ não. Em relação à atuação na área da estomaterapia, 59 (70,2\%) enfermeiros atuam na área da estomaterapia e 25 (29,8\%) não estão atuando na área em questão.

A Tabela 2 mostra que, referente à área de especialidade do estomaterapeuta atuante, 26 (31\%) trabalham com estomias e feridas; 18 (21,5\%) somente com feridas; 8 (9,5\%) com estomias, feridas e incontinências; 4 (4,7\%) com estomias e incontinências; 3 (3,5\%) somente com estomias; e nenhum participante demonstrou trabalhar somente com incontinências e feridas e incontinências especificamente.

Tabela 1. Perfil sociodemográfico dos participantes.

\begin{tabular}{|c|c|c|}
\hline Variáveis & Categorias & $\mathrm{N}^{\circ}(\%)$ \\
\hline \multirow{2}{*}{ Sexo } & Feminino & $72(85,7)$ \\
\hline & Masculino & $12(14,3)$ \\
\hline \multirow{5}{*}{ Idade (anos) } & $20-30$ & $07(8,3)$ \\
\hline & $31-40$ & $57(67,8)$ \\
\hline & $41-50$ & $11(13,1)$ \\
\hline & $51-60$ & $06(7,1)$ \\
\hline & $61-70$ & $03(3,6)$ \\
\hline \multirow{8}{*}{ Cidade onde reside } & Belo Horizonte/MG & $49(58,3)$ \\
\hline & Betim/MG & $01(1,2)$ \\
\hline & Contagem/MG & $08(9,5)$ \\
\hline & Ponta Porã/MS & $01(1,2)$ \\
\hline & São Paulo/SP & $01(1,2)$ \\
\hline & Teixeira de Freitas/BA & $01(1,2)$ \\
\hline & Vitória/ES & $01(1,2)$ \\
\hline & Interior de MG & $22(26,2)$ \\
\hline \multirow{10}{*}{ Cidade onde trabalha } & Belo Horizonte/MG & $50(59,5)$ \\
\hline & Betim/MG & $03(3,6)$ \\
\hline & Contagem/MG & $04(4,7)$ \\
\hline & Ponta Porã/MS & $01(1,2)$ \\
\hline & São Paulo/SP & $01(1,2)$ \\
\hline & Teixeira de Freitas/BA & $01(1,2)$ \\
\hline & Vitória/ES & $01(1,2)$ \\
\hline & Interior de MG & $19(22,6)$ \\
\hline & Vários estados & $02(2,4)$ \\
\hline & Desempregados & $02(2,4)$ \\
\hline \multirow{5}{*}{$\begin{array}{l}\text { Ano de conclusão do } \\
\text { curso de graduação }\end{array}$} & 1978 a 1990 & $07(8,3)$ \\
\hline & 1991 a 2000 & $07(8,3)$ \\
\hline & 2001 a 2005 & $24(28,6)$ \\
\hline & 2006 a 2010 & $36(42,8)$ \\
\hline & 2011 a 2014 & $10(11,9)$ \\
\hline \multirow{9}{*}{$\begin{array}{l}\text { Ano de conclusão do } \\
\text { curso de Estomaterapia }\end{array}$} & 2007 & $04(4,7)$ \\
\hline & 2008 & $05(6,0)$ \\
\hline & 2009 & $12(14,3)$ \\
\hline & 2010 & $10(11,9)$ \\
\hline & 2011 & $04(4,7)$ \\
\hline & 2012 & $11(13,1)$ \\
\hline & 2013 & $07(8,3)$ \\
\hline & 2014 & $12(14,3)$ \\
\hline & 2015 & $19(22,6)$ \\
\hline
\end{tabular}

Fonte: Dados da pesquisa, 2017 
Tabela 2. Perfil profissional dos participantes.

\begin{tabular}{|c|c|c|}
\hline Variáveis & Categorias & $\mathrm{N}^{\circ}(\%)$ \\
\hline \multirow{2}{*}{$\begin{array}{l}\text { Atuação como } \\
\text { enfermeiro }(n=84)\end{array}$} & Atuam & $73(86,9)$ \\
\hline & Não atuam & $11(13,1)$ \\
\hline \multirow{2}{*}{$\begin{array}{l}\text { Atuação na } \\
\text { estomaterapia } \\
(\mathrm{n}=84)\end{array}$} & $\begin{array}{l}\text { Estomaterapeutas } \\
\text { atuantes }\end{array}$ & $59(70,2)$ \\
\hline & $\begin{array}{c}\text { Estomaterapeutas não } \\
\text { atuantes }\end{array}$ & $25(29,8)$ \\
\hline \multirow{8}{*}{$\begin{array}{l}\text { Área de especialidade } \\
\text { do estomaterapeuta } \\
\text { atuante }(n=84)\end{array}$} & Estomias & $3(3,5)$ \\
\hline & Feridas & $18(21,5)$ \\
\hline & Incontinências & $0(0,0)$ \\
\hline & Estomias e feridas & $26(31,0)$ \\
\hline & $\begin{array}{c}\text { Estomias e } \\
\text { incontinências }\end{array}$ & $4(4,7)$ \\
\hline & Feridas e incontinências & $0(0,0)$ \\
\hline & $\begin{array}{l}\text { Estomias, feridas e } \\
\text { incontinências }\end{array}$ & $8(9,5)$ \\
\hline & Não atuante & $25(29,8)$ \\
\hline \multirow{10}{*}{$\begin{array}{l}{ }^{\dagger} \text { Atividades } \\
\text { relacionadas à } \\
\text { estomaterapia } \\
(n=201)\end{array}$} & Assessoria & $19(9,4)$ \\
\hline & Assistencial & $59(29,3)$ \\
\hline & Atendimento autônomo & $33(16,4)$ \\
\hline & Consultoria & $17(8,5)$ \\
\hline & Ensino & $23(11,4)$ \\
\hline & Gestão e administração & $13(6,5)$ \\
\hline & Pesquisa & $13(6,5)$ \\
\hline & Vendas & $11(5,5)$ \\
\hline & Outros & $3(1,5)$ \\
\hline & Nunca atuou & $10(5,0)$ \\
\hline \multirow{9}{*}{$\begin{array}{l}\text { Área/setor de atuação } \\
(n=190)\end{array}$} & Ambulatório & $25(13,2)$ \\
\hline & Atendimento domiciliar & $42(22,2)$ \\
\hline & Consultório privado & $9(4,7)$ \\
\hline & Hospital & $52(27,3)$ \\
\hline & Indústria & $15(7,8)$ \\
\hline & $\begin{array}{c}\text { Atenção básica (ESF - } \\
\text { UBS) }\end{array}$ & $20(10,5)$ \\
\hline & $\begin{array}{l}\text { Universidades/ } \\
\text { faculdades }\end{array}$ & $13(6,8)$ \\
\hline & Outros & $4(2,2)$ \\
\hline & Nunca atuou & $10(5,3)$ \\
\hline \multirow{4}{*}{$\begin{array}{l}\text { †Área/setor de } \\
\text { atuação (hospitalar) } \\
(n=52)\end{array}$} & Ambulatório & $9(17,0)$ \\
\hline & $\begin{array}{l}\text { Atendimento em todos } \\
\text { os setores }\end{array}$ & $27(52,0)$ \\
\hline & Terapia intensiva & $3(6,0)$ \\
\hline & Unidade de internação & $13(25,0)$ \\
\hline \multirow{4}{*}{$\begin{array}{l}{ }^{\dagger} \text { Área/setor de } \\
\text { atuação (industrial) } \\
(n=15)\end{array}$} & Comercial & $10(67,0)$ \\
\hline & Programas assistenciais & $2(13,0)$ \\
\hline & $\begin{array}{c}\text { Pesquisa e } \\
\text { desenvolvimento }\end{array}$ & $0(0,0)$ \\
\hline & científico & $3(20,0)$ \\
\hline
\end{tabular}

Tabela 2. Continuação...

\begin{tabular}{|c|c|c|}
\hline Variáveis & Categorias & $\mathrm{N}^{\circ}(\%)$ \\
\hline \multirow{4}{*}{$\begin{array}{l}\text { Tipos de serviço } \\
(n=84)\end{array}$} & Privado & $24(28,6)$ \\
\hline & Privado e público & $18(21,4)$ \\
\hline & Público & $32(38,1)$ \\
\hline & Nunca atuou & $10(11,9)$ \\
\hline \multirow{4}{*}{$\begin{array}{l}\text { Capacitações } \\
\text { pós-curso de } \\
\text { estomaterapia } \\
(n=84)\end{array}$} & $\mathrm{Sim}$ & $58(69,0)$ \\
\hline & & \\
\hline & Não & $26(31,0)$ \\
\hline & & \\
\hline \multirow{9}{*}{$\begin{array}{l}\text { †Tipos de Capacitação } \\
(n=179)\end{array}$} & Cursos & $31(17,3)$ \\
\hline & Doutorado & $1(0,7)$ \\
\hline & Mestrado & $9(5,0)$ \\
\hline & Congresso internacional & $13(7,2)$ \\
\hline & Congresso nacional & $31(17,3)$ \\
\hline & Seminários & $38(21,4)$ \\
\hline & Simpósios & $30(16,7)$ \\
\hline & Reuniões cientifica & $23(12,8)$ \\
\hline & Outros & $3(1,6)$ \\
\hline \multirow{5}{*}{$\begin{array}{l}\text { `Motivação para } \\
\text { cursar estomaterapia } \\
(n=140)\end{array}$} & Ascensão na carreira & $25(18,0)$ \\
\hline & Aumento salarial & $14(10,0)$ \\
\hline & $\begin{array}{l}\text { Conhecimento técnico } \\
\text { científico }\end{array}$ & $68(48,5)$ \\
\hline & $\begin{array}{c}\text { Atividades com paciente } \\
\text { e família }\end{array}$ & $29(20,7)$ \\
\hline & Outros & $4(2,8)$ \\
\hline
\end{tabular}

${ }^{\dagger} n \neq 8$. Fonte: Dados da pesquisa, 2017.

Das atividades relacionadas à estomaterapia, 59 $(29,3 \%)$ profissionais selecionaram a área assistencial, seguida do atendimento autônomo com $33(16,4 \%)$, da área de ensino com 23 (11,4\%), assessoria com 19 $(9,4 \%)$, consultoria com $17(8,5 \%)$, gestão/administração e pesquisa, ambas com $13(6,5 \%)$ e vendas com 11 (5,5\%), 3 (1,5\%) participantes selecionaram a opção de outras atividades, e 10 (5\%) nunca atuaram em qualquer das atividades descritas anteriormente. A questão referente a esses dados está relacionada a se o profissional exerce ou já exerceu algumas dessas atividades, sendo possível a escolha de mais de uma opção de resposta.

Os hospitais representam 52 (27,3\%) da área de atuação dos egressos do curso de Estomaterapia, sendo 27 (52\%) no atendimento a todos os setores, $13(25 \%)$ na Unidade de Internação, 9 (17\%) ambulatorial e 3 (6\%) no setor de terapia intensiva. Subsequente à área hospitalar estão 42 (22,2\%) no atendimento domiciliar, 25 (13,2\%) no ambulatório, 20 (10,5\%) na atenção básica, 15 (7,8\%) na indústria, 13 (6,8\%) nas universidades/faculdades e $9(4,7 \%)$ nos consultórios 
privados. Dos 15 (7,8\%) atuantes na indústria, 10 (67\%) estão no setor comercial, 3 (20\%) na pesquisa e desenvolvimento cientifico e 2 (13\%) nos programas assistenciais. A questão referente a esses dados está relacionada a se o profissional atua ou já atuou em alguma dessas áreas, sendo possível a escolha de mais de uma opção de resposta.

Trinta e dois $(38,1 \%)$ estomaterapeutas egressos atuam ou já atuaram no serviço público, $24(28,6 \%)$ no serviço privado, 18 (21,4\%) em ambos, e 10 (11,9\%) estomaterapeutas nunca atuaram.

Dos 84 participantes, 58 (69\%) investiram em capacitação profissional após a especialização e 26 (31\%) não. Entre os que deram continuidade na capacitação, $38(21,4 \%)$ buscaram por seminários, 31 (17,3\%) participaram de cursos e congressos nacionais, $30(16,7 \%)$ de simpósios, $23(12,8 \%)$ de reuniões científicas, 13 (7,2\%) de congressos internacionais, 9 (5\%) cursaram mestrado, $3(1,6 \%)$ realizaram outras atividades e $1(0,6 \%)$ cursou doutorado. A questão referente a esses dados está relacionada a se o profissional deu continuidade em capacitação profissional após conclusão do curso, sendo possível a escolha de mais de uma opção de resposta.

Os egressos foram questionados quanto à motivação para cursar o curso de Estomaterapia e 68 (48,5\%) justificaram pelo conhecimento técnico-científico, 29 (20,7\%) como oportunidade de desenvolver atividades com paciente e família, 25 (18\%) para ascensão na carreira, 14 (10\%) para aumento salarial e $4(2,8 \%)$ por outros motivos.

\section{DISCUSSÃO}

$\mathrm{Na}$ caracterização quanto ao sexo, prevaleceu o gênero feminino, dado corroborado pela literatura sobre o tema. $\mathrm{O}$ predomínio do gênero feminino na enfermagem é descrito por outros autores no sentido de que reproduz uma peculiaridade memorável da profissão, sendo executada em seus primórdios apenas por mulheres 5 . Dados demonstrados por pesquisa desenvolvida pelo Conselho Federal de Enfermagem (Cofen) em 2015, contabilizaram que $84,6 \%$ dos profissionais de enfermagem no Brasil é do gênero feminino e 15,4\% do gênero masculino ${ }^{6}$. Da mesma forma, esse fato é constatado em diversas outras culturas, em que o cuidado aos pacientes são apontados como continuidade da ocupação da mulher. Ademais, acredita-se ser uma característica estrutural dos serviços do setor de saúde, a superioridade das mulheres em atividades que envolvem a assistência às pessoas ${ }^{7-10}$.
Dada a maioria do sexo feminino entre os profissionais desta amostra, verificou-se ser este um cenário muito comum em estudos realizados em outros locais: entre os egressos do curso de graduação em Enfermagem da Universidade Estadual de Londrina (UEL), 90\% são do sexo feminino; em unidades de terapia intensiva (UTI) no Brasil, 82,5\% são enfermeiras; entre enfermeiros gerentes de hospital de ensino em Ribeirão Preto, 93,3\% são do sexo feminino; em participantes do curso de aprimoramento para Enfermagem Obstétrica da Universidade Federal de Minas Gerais (UFMG), a maioria era do sexo feminino $(89 \%)^{8,11-14}$. Em estudo que traçou o perfil do estomaterapeuta egresso da Universidade de Taubaté, foi identificada a predominância de especialistas em estomaterapia do gênero feminino $(29 / 96,6 \%)^{15}$. Esses fatos corroboram os dados desta pesquisa. Entretanto, é importante sublinhar que já é notório o crescimento da representatividade masculina na enfermagem e na especialidade, de acordo com resultados obtidos neste estudo.

Em relação à idade, a maioria das pessoas apresentaram idade entre 31 a 40 anos, dado corroborado por estudos em que os participantes tinham idade entre 20 e 50 anos, período em que ocorre a busca pelo delineamento ou concretização do desenvolvimento profissional ${ }^{8,16}$. No estudo realizado na Universidade de Taubaté ${ }^{15}$, também foi encontrado um valor significativo (15/50\%) de egressos com idade de 36 a 50 anos. Em outro estudo, que tinha como objetivos discutir e analisar o perfil dos enfermeiros de uma UTI, a média de idade foi de 39,4 anos, com amplitude mínima de 29 anos e máxima de 60 anos $^{10}$. Dados que reforçam um perfil de profissional com um período mínimo de experiência na área assistencial ou a busca do retorno de atividades acadêmicas em um período de maior estabilidade e maturidade em sua carreira profissiona ${ }^{15}$.

As oportunidades de trabalho nos grandes centros e as facilidades de acesso ao curso de Estomaterapia justificam o percentual de 58\% dos egressos residentes em Belo Horizonte/ MG, local onde o curso é oferecido, e $26 \%$ residentes em cidades do interior de Minas Gerais. Em estudo realizado com os egressos da Universidade de Taubaté, a maioria (23/76,6\%) dos entrevistados residia na grande São Paulo ${ }^{15}$.

Do total de egressos da UFMG,22 (26,2\%) são residentes em cidades do interior de Minas Gerais. Esse dado traduz coerência com uma das propostas do curso de especialização em Enfermagem em Estomaterapia da instituição em estudo, que é produzir impacto social, por meio da disseminação do conhecimento científico da especialidade para as regiões 
com menor representação dos profissionais especialistas e serviços especializados da área, propiciando melhor assistência às pessoas que necessitam da assistência do estomaterapeuta. Essa dispersão está em conformidade com um dos princípios deste curso, ou seja, a preocupação em selecionar proporcionalmente candidatos de outras regiões de Minas Gerais e do Brasil, com vistas à disseminação do conhecimento e de especialistas por todo o país ${ }^{2,15,16}$.

Estudo realizado por Martins e colaboradores (2006) identificou que o tempo de conclusão do curso pode ser um indício do tempo de vivência do profissional no mercado de trabalho e de concernente maturidade ${ }^{7}$. O bacharelado revela as competências e habilidades do enfermeiro, assim como o tempo de formação em uma dada época reflete o conhecimento e a aptidão valorizados em um determinado período ${ }^{17}$. Nesta pesquisa, predominou o número de concluintes no curso de graduação entre os anos de 2006 a 2010, correspondendo a 36 (42,8\%) profissionais.

Dos 84 participantes desta pesquisa, a predominância do ano de conclusão do curso de Estomaterapia foi 2015, quando houve 19 (22,7\%) concluintes, e do ano de 2014, quando houve 12 (14,2\%) concluintes. Tais resultados podem ter sido influenciados pela atualização mais recente dos dados de contato dos participantes com o ano de conclusão do curso próximo ao ano da pesquisa.

A especialidade em estomaterapia está em crescimento no mercado nacional por ter uma ampla possibilidade de atuação profissional, em assistência direta ao paciente, ensino, pesquisa, administração, vendas, assessoria e consultoria. A necessidade de novos profissionais de enfermagem qualificados para atuarem nos serviços é uma realidade. $\mathrm{O}$ enfermeiro e o estomaterapeuta são profissionais liberais e podem atuar em serviços de saúde das redes pública e privada, ambulatórios, clínicas, consultórios especializados em estomaterapia, assistência domiciliar e têm a possibilidade de empreender seu próprio negócio ${ }^{3,18}$.

Deve-se destacar que este estudo identificou que o mercado de trabalho para os estomaterapeutas tem absorvido os especialistas com relativo sucesso, considerando que, dos 84 participantes da pesquisa, 73 (87\%) atuam como enfermeiros e 59 (70\%) na área de estomaterapia. Esse resultado corrobora o que se tem observado na prática, que o curso de especialização em Enfermagem em Estomaterapia tem sempre crescente procura. Não se pode também esquecer que outros fatores são atrativos para essa crescente busca pelo curso, tais como o reconhecimento de sua qualidade por profissionais de excelência, a participação de estomaterapeutas renomados nas disciplinas específicas, o reconhecimento formal e legal do curso pela Sobest, pelo Ministério da Educação e Cultura (MEC), pela própria UFMG e por instituições nacionais e internacionais da área ${ }^{19}$.

Em um estudo sobre o perfil sociodemográfico dos enfermeiros da rede hospitalar, constatou-se que os altos índices de profissionais realizando cursos de especialização $(65,4 \%)$ deve-se ao perfil de profissional desejado pelo mercado, com aprofundamento técnico-científico dentro da área específica de atuação. Enfermeiros que possuem somente o curso de graduação, por atuarem como generalistas, são pouco desejados pelo mercado e estão mais expostos às dificuldades na atividade profissional ${ }^{17}$.

Em estudo realizado por Paula e Santos ${ }^{20}$, a falta de reconhecimento institucional foi apontada como aspecto negativo, pois colabora para a desvalorização do estomaterapeuta, em razão da falta de interesse e compreensão das atividades especializadas, bem como da limitada relevância atribuída às funções realizadas e incompreensão dos desfechos efetivos e assertivos dele advindos. Sendo assim, refere-se a mais uma provocação para o estomaterapeuta: poder colaborar para a ressignificação de seu valor e para o reconhecimento da especialidade na sociedade. Tais afirmações podem ser uma possível justificativa aos resultados da pesquisa, que informa que $13 \%$ dos egressos não atuam como enfermeiros e 30\% não atuam na área da especialidade. Entretanto, a especialidade é considerada nova e em grande crescimento no mercado de trabalho.

Em um estudo realizado na área da obstetrícia, também pode-se perceber dificuldade de inserção dos especialistas em enfermagem obstétrica, por se sentirem de forma inautêntica e imprópria sobre sua atuação prática, sem identidade profissional, com acúmulo ou desvio de função, muitas vezes excluídos da equipe de saúde, o que gera estado de conformismo ou frustração. Tais dificuldades podem ser reflexo dos resultados negativos de abandono da profissão e não atuação na especialidade ${ }^{8}$.

No que tange à área de atuação da estomaterapia, os resultados obtidos mostraram que os egressos do curso, em sua maioria, trabalham com estomias e feridas; seguido por somente com feridas; com estomias, feridas e incontinências; com estomias e incontinências; e somente com estomias. Nenhum participante informou trabalhar especificamente e apenas com incontinências ou feridas ou incontinências. Ressalta-se nos dados obtidos o baixo percentual de 
estomaterapeutas que têm se dedicado às incontinências e, mesmo assim, aliadas à atuação com estomias. Tais dados revelam uma escassez de profissionais atuando na área de incontinências e, consequentemente, um reduzido quantitativo de serviços que atendam nessa área.

O estomaterapeuta, quando vinculado profissionalmente a uma instituição de saúde, a cooperativas de grupo ou sendo efetivo trabalhador liberal, possui suporte legal para exercer funções assistenciais, educativas, de pesquisa e assessoria ${ }^{15}$. Existe uma ampla diversidade de campos de atuação para os estomaterapeutas, que se percebe crescente no mercado de saúde no Brasil, onde cada vez mais aportam empresas com insumos e tecnologias inovadoras que necessitam desses especialistas para testar e avaliar cientificamente seus produtos, analisar a relação custo-benefício para os pacientes e instituições, indicar e divulgar suas produções. Considerando também esse fator, percebe-se que ele pode fomentar e instigar a procura pela especialização em estomaterapia ${ }^{19}$.

No que se refere à área de atuação dos egressos participantes deste estudo, percebeu-se que, das atividades relacionadas à estomaterapia, foi predominante a área assistencial 59 (29,3\%), seguida de atuação autônoma 33 $(16,4 \%)$, atividades de ensino 23 (11,4\%), assessoria 19 $(9,4 \%)$, consultoria $17(8,5 \%)$, gestão/administração e pesquisa 13 (6,5\%) e vendas 11 (5,5\%). Observa-se uma predominância de atuação dos egressos na área assistencial, que pode estar relacionada às oportunidades de emprego no mercado de trabalho, decorrente de uma necessidade de cuidado especializado e aumento da valorização da prática em estomaterapia na assistência prestada pelos estomaterapeutas. Já a prática de atuação autônoma está em crescimento na área da enfermagem, com apoio das legislações vigentes, pela busca dos enfermeiros por independência do atual mercado de trabalho competitivo, devido à crise financeira nos serviços de saúde ${ }^{21}$.

Em estudo realizado em Portugal, foi salientado que a importância dos cuidados do enfermeiro, no âmbito da consulta de enfermagem de estomaterapia, impacta positivamente nos processos de transição do paciente que, devido às grandes transformações pessoais, requer aceitação e adaptação à nova condição de vida ${ }^{22}$. Esse contexto reflete as diversas oportunidades de atuação do enfermeiro estomaterapeuta que exerce diferentes funções no exercício da estomaterapia nos aspectos preventivos, terapêuticos e de reabilitação em busca da melhoria da qualidade de vida dos pacientes ${ }^{3}$.
Por meio dos resultados deste estudo, foi possível identificar que os hospitais representam 27,3\% da área de atuação dos egressos do curso de estomaterapia da UFMG. Desses $27,3 \%, 52 \%$ equivale ao atendimento a todos os setores, $25 \%$ na unidade de internação, $17 \%$ em ambulatório, $6 \%$ no setor de terapia intensiva. Esses dados corroboram estudo que abrange a estomaterapia no Brasil no período de 1990 a 1995, em que 58\% dos respondentes também atuavam em unidades hospitalares ${ }^{16}$. Em relação à área de atuação de enfermeiros especialistas, outro estudo também apresentou predomínio (93,60\%) na assistência hospitalar ${ }^{8}$.

O percentual das áreas de atuação subsequentes à área hospitalar foi assim distribuído: 22,2\% em atendimento domiciliar, 13,2\% em ambulatório, 10,5\% em atenção básica, 7,8\% na indústria, 6,8\% em universidades/faculdades e 4,7\% em consultórios privados. Esses dados apresentam-se contrários aos resultados obtidos por Santos ${ }^{16}$, em que a segunda maior área de atuação dos estomaterapeutas era em ambulatórios, enquanto o presente estudo apresenta outra realidade, em que há direcionamento dos profissionais para $\mathrm{o}$ atendimento domiciliar.

O resultado de 27,3\% de atuação em âmbito hospitalar, predominante neste estudo, é descrito em crescimento no estudo de Teixeira e colaboradores publicado em $2016^{19}$, que relata que a instituição de um serviço de estomaterapia na área hospitalar tem o objetivo de aprimorar e ampliar a assistência aos pacientes internos ou desospitalizados, com atualizações na assistência utilizando terapêuticas eficientes e excelência no cuidado, além da diminuição de custos e do tempo de internação hospitalar.

$\mathrm{O}$ atendimento domiciliar com um resultado de 22,2\% de atuação dos egressos está provavelmente inserido na forma autônoma de trabalho do enfermeiro. De acordo com o estudo de Kraemer e colaboradores ${ }^{21}$, o enfermeiro autônomo tem a capacidade de dar seguimento em sua prática profissional, ciente de suas áreas de atuação, busca contentamento próprio e dos seus pacientes, considerando a relevância que sua atuação representa para as pessoas, os processos de trabalho e os serviços de saúde.

$\mathrm{Na}$ percepção dos profissionais, é ressaltada a atenção domiciliar como modalidade inovadora e humanizada de serviço, alternativa ao modelo de saúde hospitalocêntrico, evidenciando-se, ainda, seu caráter interdisciplinar ${ }^{22}$.

Andrade e colaboradores ${ }^{23}$ demostraram que a atuação do enfermeiro no espaço domiciliar é fundamental e ampla, 
destacando ações relacionais e educacionais, sendo necessárias inclusive nos cuidados técnicos.

Para o desempenho dessa autonomia, é essencial que o mercado de trabalho reflita as mudanças que vêm acontecendo com a atuação do enfermeiro no mundo e possibilite que ele exerça sua capacidade imaginativa e de resolução ${ }^{21}$.

A realidade no cenário hospitalar e na atenção primária à saúde deixaram de ser as principais fontes de empregabilidade e afastam o profissional para diversas oportunidades em que ele experimenta maior independência ${ }^{15}$.

No que tange à atuação atual ou anterior dos participantes da pesquisa em serviços públicos ou privados, obteve-se que 32 (38\%) estomaterapeutas egressos atuam ou já atuaram no serviço público, seguido de 24 (29\%) no serviço privado e 18 (21\%) em ambos. Em um estudo recente ${ }^{8}$, muitos egressos do curso de especialização em Enfermagem Obstétrica $(65,6 \%)$ também trabalhavam em serviços públicos. Outro estudo corrobora os dados apresentados, de que a maioria (49\%) dos enfermeiros da rede hospitalar possui vínculo empregatício em instituições públicas ${ }^{17}$. Tais dados podem ser consequência de melhor estruturação de políticas em saúde em favor dos pacientes atendidos pela rede pública, o que aumenta as oportunidades no mercado de trabalho público para os enfermeiros ${ }^{4}$.

De acordo com os resultados, dos 84 participantes, 58 (69\%) investiram em capacitação profissional após a especialização e 26 (31\%) não. Entre os profissionais que deram continuidade na capacitação, $38(21,4 \%)$ buscaram por seminários, 31 (17,3\%) participaram de cursos e congressos nacionais, 30 (16,7\%) de simpósios, 23 (12,8\%) de reuniões científicas, 13 (7,2\%) de congressos internacionais, 9 (5\%) cursaram mestrado, 3 (1,6\%) realizaram outras atividades e $1(0,6 \%)$ cursou doutorado. Lembrando que nessa questão foi possível a escolha de mais de uma opção de resposta.

Outro estudo aponta que $25,86 \%$ dos egressos do curso em Enfermagem Obstétrica cursaram mestrado, 3,45\% doutorado e 76,86\% participaram de congressos e eventos na área da obstetrícia ${ }^{8}$, o que demonstra maior investimento no processo de formação do profissional pós-especialização.

Procurar cursos na área da especialidade é ansiar por mestria na sua atuação, movimentando e unindo conhecimentos, colocando valores e competências em execução, a fim de alcançar eficiência na realização de tarefas requisitadas pela natureza do trabalho e para resolução dos problemas e situações imprevisíveis em um cenário cultural ${ }^{8}$. A procura contínua e imprescindível de qualificação técnica também foi ressaltada pelos estomaterapeutas, apresentando-se como questão essencial para a elaboração da identidade profissional do enfermeiro, colaborando ainda, com vigor e positivamente, para a atualização e o desenvolvimento científico $^{20}$.

Os 31\% dos egressos que não realizaram nenhum tipo de capacitação pós-conclusão do curso podem apresentar dificuldades de inserção ou de manutenção no mercado de trabalho específico, visto que a continuidade da busca de conhecimento na área tem que ser constante ${ }^{20}$.

Entre os motivos para cursar a especialização em estomaterapia, os egressos responderam, em sua maioria, o interesse por conhecimento técnico-científico (48,5\%), seguido por oportunidade de desenvolver atividades com paciente e família (20,7\%), ascensão na carreira (18\%), aumento salarial (10\%) e outros motivos (2,8\%). Segundo Dias e colaboradores ${ }^{15}$, cursos de pós-graduação podem ser considerados estratégias para os requisitos da empregabilidade de profissionais competentes, com independência para tomada de decisões, para introduzir avanços tecnológicos e responsabilizar-se por contestar as adversidades dos mais diversos meios de produção, o que corrobora o interesse da maioria dos egressos do curso de estomaterapia da UFMG.

Com relação às limitações desta pesquisa, foi possível perceber que há escassez de publicações nacionais e ausência de estudos internacionais acerca da temática, o que dificultou a discussão sobre os resultados apresentados. Assim, faz-se necessário ampliar as pesquisas sobre a atuação do estomaterapeuta.

Os resultados deste estudo contribuirão para o dimensionamento e mapeamento da inserção dos estomaterapeutas no mercado de trabalho e a possibilidade de avaliar as oportunidades de absorção da prática especializada do estomaterapeuta.

\section{CONCLUSÃO}

Este estudo possibilitou a identificação da área de atuação, trajetória na especialidade e posteriores capacitações como especialista na área, dos estomaterapeutas egressos da Universidade Federal de Minas Gerais.

Os enfermeiros estomaterapeutas egressos eram, em sua maioria, do sexo feminino, com idade entre 31 e 40 anos, residentes e atuantes no mercado de trabalho de 
Belo Horizonte/MG. Mantêm-se atuantes na área de especialidade, com predominância dos cuidados às estomias e feridas, atividades assistenciais, na área hospitalar e no setor público de saúde. A busca pela especialização foi motivada principalmente pelo interesse no desenvolvimento técnico-cientifico e os egressos permanecem dando continuidade nas capacitações após a realização da especialidade.

A estomaterapia encontra-se em expansão e em grande crescimento no cenário nacional, sendo essencial o seguimento de pesquisas dessa esfera para uma visão mais abrangente da especialidade e intervenções da Associação Brasileira de Estomaterapia para contínua consolidação da estomaterapia no Brasil.

\section{AGRADECIMENTOS}

Agradecemos a todos os estomaterapeutas participantes da pesquisa realizada neste trabalho.

\section{CONTRIBUIÇÃO DOS AUTORES}

Conceitualização, Gontijo TG; Borges EL e Ferraz AF; Metodologia, Gontijo TG; Borges EL; Ferraz AF e Pires Júnior JF; Investigação, Gontijo TG; Redação - Primeira versão, Gontijo TG; Borges EL e Ferraz AF; Redação Revisão \& Edição, Gontijo TG; Borges EL; Pires Júnior JF e Spira JAO; Supervisão, Borges EL.

\section{REFERÊNCIAS}

1. Boyle DK. Nursing Specialty Certification and Patient Outcomes: What We Know in Acute Care Hospitals and Future Directions. J Assoc Vasc Access. 2017;22(3):137-42. https://doi.org/10.1016/j.java.2017.06.002

2. Yamada BF, Ferrola EC, Azevedo GR, Blanes L, Rogenski NM, Santos VL. Competências do Enfermeiro Estomaterapeuta (ET) ou do Enfermeiro Pós-graduado em Estomaterapia (PGET). ESTIMA, Braz J Enterostomal Ther. 2008;6(1):33-43.

3. SteltonS. TheWCET at40.Adv Skin Wound Care. 2018;31(4):15051. https://doi.org/10.1097/01.ASW.0000531296.56431.c1

4. Santos VLCG. Cuidando do estomizado: análise da trajetória no ensino, pesquisa e extensão. São Paulo. Tese (LivreDocência) - Escola de Enfermagem da Universidade de São Paulo; 2006.

5. Viana RAPP, Vargas MAO, Carmagnani MIS, Tanaka LH, Luz KR, SchmittPH. Perfil do enfermeiro de terapia intensiva em diferentes regiões do Brasil. Texto Contexto Enferm. 2014;23(1):151-59. https://doi.org/10.1590/S0104-07072014000100018

6. Conselho Federal de Enfermagem (Cofen). Pesquisa inédita traça perfil da enfermagem [internet]. [Citado 18 dez 2018]. Disponível em: http://www.cofen.gov.br/pesquisa-ineditatraca-perfil-da-enfermagem_31258.html

7. Martins C, Kobayashi RM, Ayoub AC, Leite MMJ. Perfil do enfermeiro e necessidades de desenvolvimento de competência profissional. Texto Contexto Enferm. 2006;15(3):472-78. https:// doi.org/10.1590/S0104-07072006000300012

8. Carlos GAV, Matozinhos FP, Carmo JMA, Manzo BF, Duarte ED, Souza KV. Perfil dos participantes de um curso de aprimoramento em Enfermagem obstétrica. REME - Rev Min Enferm. 2019;23:e-1153. https://doi.org/10.5935/14152762.20190006
9. Nicácio MC, Heringer ALS, Schroeter MS, Pereira ALF. A prática profissional das enfermeiras obstétricas na casa de parto e hospital: um estudo descritivo. Online Braz J Nurs. 2016;15(2):205-14. https://doi.org/10.17665/16764285.20165203

10. Oliveira PVN, Matias AO, Valente GSC, Messias CM, Rosa FSMS, Souza JDF. Formação do enfermeiro para os cuidados de pacientes críticos na Unidade de Terapia Intensiva. Nurs. 22(250):2751-2755.

11. Camelo SHH, Soares MI, Chaves LDP, Rocha FLR, Silva VLS. Nurse managers atateaching hospital:training, responsibilities and challenges. Rev Enferm UERJ. 2016;24(3):1-6. https://doi. org/10.12957/reuerj.2016.11637

12. Souza SNDH, Miyadahira AMZ. O desenvolvimento de competências no Curso de Graduação em Enfermagem de egressos. Cienc Cuid Saúde. 2012;1:243-50. https://doi. org/10.4025/cienccuidsaude.v11i5.17082

13. Cavalcante EFO, Silva DMGV. Nurses' commitment to the care of tuberculosis patients. Texto Contexto Enferm. 2016;25(3):110. https://doi.org/10.1590/0104-07072016003930015

14. Lazarini FM, Barbosa DA. Educational intervention in Primary Care for the prevention of congenital syphilis. Rev Latino-Am Enfermagem. 2017;25:1-9. https://doi.org/10.1590/15188345.1612.2845

15. Dias MSC, Boccara de Paula MA, Morita ABPS. Perfil Profissional de Enfermeiros Estomaterapeutas Egressos da Universidade de Taubaté. ESTIMA, Braz J Enterostomal Ther. 2014;12(3).

16. Santos VLCG. Ensino especializado de estomaterapia no Brasil:1990-1995. Rev Latino-Am Enfermagem. 1998;6(3):4354. https://doi.org/10.1590/S0104-11691998000300006 
17. Araujo MAN, Lunardi Filho WD, Alvarenga MRM, Oliveira RD, Souza JC, Vidmantas S. Perfil sociodemográfico dos enfermeiros da rede hospitalar. Rev Enferm UFPE. 2017;11(11):4716-725.

18. Fernandes JD, Silva RMO, Mota LSR, Silva ACP, Silva LS, Freitas CM. Mapeamento dos Cursos de Especialização em enfermagem em sua totalidade e contradições. Rev Enferm UFPE. 2017;11(6):2459-465.

19. Teixeira AS, Menezes LC, Oliveira RM. Serviço de Estomaterapia na perspectiva dos gerentes de enfermagem em Hospital Público de Referência. ESTIMA, Braz J Enterostomal Ther. 2016;14(1).

20. Paula MAB, Santos VLCG. O significado de ser especialista para o enfermeiro estomaterapeuta. Rev Latino-Am
Enfermagem. 2003;11(4):474-82. https://doi.org/10.1590/ S0104-11692003000400010

21. Kraemer FZ, Duarte MLC, Kaiser DE. Autonomia e trabalho do enfermeiro. Rev Gaúcha Enferm. 2011;32(3):487-97. https://doi.org/10.1590/S1983-14472011000300008

22. Miranda LSG, Carvalho AAS, Paz EPA. Qualidade de vida da pessoa estomizada: relação com os cuidados prestados na consulta de enfermagem de estomaterapia. Esc Anna Nery. 2018;22(4):487-94. https://doi.org/10.1590/2177-9465ean-2018-0075

23. Andrade $A M$, Silva $K L$, Seixas $C T$, Braga PP. Atuação do enfermeiro na atenção domiciliar: uma revisão integrativa da literatura. Rev Bras Enferm. 2017;70(1):210-19. https:// doi.org/10.1590/0034-7167-2016-0214 